A. Ragusa and G. Zappalà

Nagoya Math. J.

Vol. 162 (2001), 111-125

\title{
GORENSTEIN SCHEMES ON GENERAL HYPERSURFACES OF $\mathbb{P}^{r}$
}

\author{
ALFIO RAGUSA AND GIUSEPPE ZAPPALÀ
}

\begin{abstract}
It is completely known the characterization of all Hilbert functions and all graded Betti numbers for 3-codimensional arithmetically Gorenstein subschemes of $\mathbb{P}^{r}$ (works of Stanley [St] and Diesel [Di]). In this paper we want to study how geometrical information on the hypersurfaces of minimal degree containing such schemes affect both their Hilbert functions and graded Betti numbers. We concentrate mainly on the case of general hypersurfaces and of irreducible hypersurfaces, for which we find strong restrictions for the Hilbert functions and graded Betti numbers of their subschemes.
\end{abstract}

\section{Introduction}

The paper of Stanley [St] completely settled the question about all the possible Hilbert functions for 3-codimensional arithmetically Gorenstein subschemes of $\mathbb{P}^{r}$. Precisely, $H$ is the Hilbert function of a 3-codimensional arithmetically Gorenstein subscheme of $\mathbb{P}^{r}$ if and only if $\Delta^{r-2} H$ is "symmetric" and the "first half" of $\Delta^{r-1} H$ is an $O$-sequence. Similarly, the paper of Diesel [Di] settled the question about all the possible graded Betti numbers of this kind of subschemes. Precisely, the positive integers $d_{0} \leq d_{1} \leq \cdots \leq d_{2 m}$ are generators degrees of a 3 -codimensional arithmetically Gorenstein subscheme of $\mathbb{P}^{r}$ if and only if $\sum_{0 \leq i \leq 2 m} d_{i} / m=\vartheta$ is an integer and $\vartheta>d_{i}+d_{2 m+1-i}$ for every $i=1, \ldots, m$. In this case the degrees of the first syzygies are $\vartheta-d_{i}$ for $i=0,1, \ldots, 2 m$, and the only second syzygy has degree $\vartheta$.

Many other papers recently investigate the Hilbert functions and the graded Betti numbers of 3-codimensional arithmetically Gorenstein subschemes of $\mathbb{P}^{r}$, mainly based on the structure theorem of Buchsbaum and Eisenbud, see for instance [DV], [GM], [Ha], [HTV], [IK], [RZ], [Ul].

In this paper, continuing the work started in [RZ], we want to study how the "geometry" of the hypersurfaces of minimal degree containing a 3-

Received November 2, 1999.

1991 Mathematics Subject Classification: 13D40, 13H10. 
codimensional arithmetically Gorenstein subscheme of $\mathbb{P}^{r}$ affects its Hilbert function and its graded Betti numbers. Of course, it seems natural to start this investigation looking at the more general case: indeed, since in $\mathbb{P}^{3}$ the generic surface $S$ of degree $\geq 4$ has $\operatorname{Pic}(S)=\mathbb{Z}$ and in $\mathbb{P}^{r}, r \geq 4$, every nonsingular hypersurface $S$ has $\operatorname{Pic}(S)=\mathbb{Z}$ (generated by the hyperplane section), we focus our attention to those 3-codimensional arithmetically Gorenstein subschemes of $\mathbb{P}^{r}$ contained in hypersurfaces of minimal degree with the Picard group equal to $\mathbb{Z}$. Of course this forces $S$ from one side to be irreducible and on the other side to have "too few" divisors: on $S$ are not allowed 2-codimensional subschemes of $\mathbb{P}^{r}$ other than the complete intersection of $S$ with another hypersurface. This is, in some sense, our key point to give restrictions on the Hilbert functions and on the graded Betti numbers for arithmetically Gorenstein subschemes of such hypersurfaces.

We still strongly make use of the Buchsbaum-Eisenbud structure theorem (see $[\mathrm{BE}]$ ) analyzing the relationships between the geometry of a 3codimensional arithmetically Gorenstein subscheme $X$ of $\mathbb{P}^{r}$ and an alternating matrix associated to it.

After notation and preliminaries we construct on any hypersurface of $\mathbb{P}^{r}$ non-trivial 3-codimensional arithmetically Gorenstein subschemes. This allows us to give a negative answer (Example 2.1) to a question posed by Geramita and Migliore in [GM]: is any 0-dimensional arithmetically Gorenstein scheme of $\mathbb{P}^{3}$ the intersection of two arithmetically Cohen-Macaulay curves linked in a complete intersection?

In Section 3 we will find some restrictions on the Hilbert functions of a 3-codimensional arithmetically Gorenstein scheme $X$, contained in a general hypersurface of minimal degree of $\mathbb{P}^{r}$. Precisely if $\Delta^{r} H_{X}(t+1)=-1$ for some $t+1 \leq \min \left\{i \in \mathbb{N} \mid \Delta^{r-1} H_{X}(i) \leq 0\right\}$ then $\Delta^{r} H_{X}(t) \geq-1$ (Corollary 3.4).

Section 4 is devoted to find restrictions on the graded Betti numbers for these schemes; the results are summarized in Corollary 4.8: if $m_{n}, M_{n}$ denote respectively the minimum and the maximum number of generators of degree $n$ for a 3-codimensional arithmetically Gorenstein scheme with a fixed Hilbert function $H$, if we know that the hypersurface of minimal degree containing the scheme $X$ is general then

$$
\left\{\begin{array}{l}
M_{n} \leq m_{n}+1 \Longrightarrow \alpha_{n}=m_{n} \\
M_{n} \geq m_{n}+2 \Longrightarrow m_{n} \leq \alpha_{n} \leq M_{n}-2 .
\end{array}\right.
$$

It seems interesting at this point to find 3-codimensional arithmetically 
Gorenstein schemes in $\mathbb{P}^{r}$ for which $\alpha_{n}>m_{n}$ for some $n$ and lying on a general hypersurface of minimal degree.

\section{$\S 1$. Notation and preliminaries}

Throughout this paper $k$ will denote an algebraically closed field, $\mathbb{P}^{r}$ the $r$-dimensional projective space over $k$,

$$
R=k\left[x_{0}, x_{1}, \ldots, x_{r}\right]=\bigoplus_{n \in \mathbb{Z}} H^{0}\left(\mathscr{O}_{\mathbb{P}^{r}}(n)\right) .
$$

We recall that if $X \subset \mathbb{P}^{r}, r \geq 3$, is a 3-codimensional arithmetically Gorenstein $(\mathrm{aG})$ scheme then its Hilbert function $H$ looks like

\begin{tabular}{c|ccccccccccccc}
$n$ & 0 & 1 & $\cdots$ & $\gamma$ & $\gamma+1$ & $\cdots$ & $\delta-1$ & $\delta$ & $\cdots$ & $\vartheta-3$ & $\vartheta-2$ & $\vartheta-1$ & $\cdots$ \\
\hline$\Delta^{r-1} H$ & 1 & 2 & $\cdots$ & $a$ & 0 & $\cdots$ & 0 & $-a$ & $\cdots$ & -2 & -1 & 0 & $\rightarrow$
\end{tabular}

where the sequence $\{1,2, \ldots, a, 0, \rightarrow\}$ is an $O$-sequence, $\Delta^{r-1} H$ is antisymmetric, i.e. $\Delta^{r-1} H(i)=-\Delta^{r-1} H(\vartheta-2-i)$ (see [St]).

In the sequel for a 3 -codimensional aG scheme $X \subset \mathbb{P}^{r}$ with Hilbert function $H$ we will use the following notation:

$$
\begin{aligned}
& \vartheta:=\max \left\{i \in \mathbb{N} \mid \Delta^{r+1} H(i) \neq 0\right\} \\
& u:=\min \left\{i \in \mathbb{N} \mid \Delta^{r-1} H(i)<0\right\}-\max \left\{i \in \mathbb{N} \mid \Delta^{r-1} H(i)>0\right\}-1
\end{aligned}
$$

Note that $u$ is just the number of zeros "in the middle" of $\Delta^{r-1} H$.

Moreover such a scheme $X$ will have a graded minimal free resolution of the following type

$$
0 \longrightarrow R(-\vartheta) \longrightarrow \bigoplus_{i} R(-i)^{\beta_{i}} \longrightarrow \bigoplus_{i} R(-i)^{\alpha_{i}} \longrightarrow I_{X} \longrightarrow 0
$$

so, in particular, $\alpha_{i}$ will denote the number of minimal generators for $I_{X}$ in degree $i$ and $\beta_{i}$ will be the number of minimal generators in degree $i$ for the first syzygies module for $I_{X}$.

Recall that, by results of Buchsbaum and Eisenbud (see [BE]), a set of minimal generators for $I_{X}$ consists of the pfaffians of an alternating matrix of odd order $2 m+1$. In particular, the minimal number of generators for $I_{X}$ must be odd. Moreover if $d_{0} \leq d_{1} \leq \cdots \leq d_{2 m}$ are the degrees of a minimal set of generators of $I_{X}$, then $\vartheta=\sum_{i=0, \ldots, 2 m} d_{i} / m$ and the syzygies degrees are given by $\vartheta-d_{i}$ for $i=0, \ldots, 2 m$. 
Our purpose in this paper is to study 3-codimensional aG schemes on general hypersurfaces of $\mathbb{P}^{r}$, which means hypersurfaces $S$ such that $\operatorname{Pic}(S)=\mathbb{Z}$. Recall that for $r \geq 4$ all nonsingular hypersurfaces are general in our meaning and for $r=3$ the generic surface of degree $d \geq 4$ is general. In particular, on such a general hypersurface $S$ the only divisors are the complete intersections $S \cap T$ for some hypersurface $T$.

\section{$\S 2$. Construction of aG schemes on general hypersurfaces}

Let $S \subset \mathbb{P}^{r}$ be a general hypersurface; a very first question is to ask if in such an hypersurface there are 3-codimensional aG schemes which are not complete intersection. The following construction will permit to build on any given hypersurface $S$ non-trivial aG schemes.

Let $S \subset \mathbb{P}^{r}$ be any hypersurface, $I_{S}=(f)$, and consider on it a 3codimensional aG scheme $X$ such that $f$ is not a minimal generator for $I_{X}$. If we perform two suitable linkages we can obtain a subscheme $X^{\prime}$ on $S$ which is still arithmetically Gorenstein: precisely it is enough to use first a complete intersection containing $X$ using $S$ and two more forms of which at least one is not a minimal generators for $I_{X}$; we obtain a linked scheme $Y$ having $f$ as a minimal generator. Now we perform another linkage using a complete intersection $(f, g, h)$, containing $Y$, where $g$ and $h$ are two more minimal generators of $I_{Y}$ (note that $I_{Y}$ has just four minimal generators).

Observe that with this construction, if we start from an aG scheme $X$ with $\nu\left(I_{X}\right)=n$ then the scheme $X^{\prime}$ will have $\nu\left(I_{X^{\prime}}\right)=n+2$. In particular on our hypersurface $S$, starting from "small" complete intersection we get aG schemes with five generators.

Now we will use the previous construction to find a counterexample to the following question posed by Geramita and Migliore in [GM]: is any 0dimensional aG subscheme of $\mathbb{P}^{3}$ the intersection of two aCM curves linked in a complete intersection?

EXAmple 2.1. Consider a general quartic surface $S$ of $\mathbb{P}^{3}, I_{S}=(f)$ and $X=\{P\}$ a subscheme of $S$ consisting just of one point. Let $Y$ be the scheme linked to $X$ in a complete intersection of type $(1,4, a), a>4$, using $S$. Observe that $I_{Y}$ is generated by four forms of degree 1, 4, $a, a+2$. Again let $X^{\prime}$ be the scheme linked to $Y$ in a complete intersection of type $(4, a, a+2)$, using $S$; as we said in the previous construction, $X^{\prime}$ will be an aG scheme whose minimal graded free resolution looks like

$$
0 \longrightarrow R(-2 a-5) \longrightarrow R(-2 a-1) \oplus R(-a-5) \oplus R(-a-3)^{3} \longrightarrow
$$


$\longrightarrow R(-4) \oplus R(-a) \oplus R(-a-2)^{3} \longrightarrow I_{X^{\prime}} \longrightarrow 0$.

Now we will show that do not exist two aCM curves $C, D$ linked in a complete intersection of type $(h, k)$ such that $C \cap D=X^{\prime}$. Suppose that $I_{X^{\prime}}=I_{C}+I_{D}$ for some curves $C$ and $D$. Since $S$ is the only generator of minimal degree for $I_{X^{\prime}}$ it must be generator for $I_{C}$ or $I_{D}$, say $f \in I_{C}$. By the "generality" of $S, C$ should be a complete intersection $S \cap T$. Since $D$ is linked to $C, I_{D}$ is minimally generated by three elements, therefore, as $\nu\left(I_{X^{\prime}}\right)=5$ there are only two possible cases: or $\operatorname{deg} T=a$ or $\operatorname{deg} T=a+2$; in the first case $I_{D}$ should be generated by three elements of degree $a+2$ and in the second case by an element of degree $a$ and two of degree $a+2$. In both cases one easily shows that $C$ and $D$ cannot be linked in a complete intersection.

Of course analogous examples can be reproduced in any $\mathbb{P}^{r}$.

\section{$\S 3$. Hilbert functions on general hypersurfaces}

Despite of the fact that on a general hypersurface $S$ one can build lots of aG schemes which are not complete intersections, for instance using the construction as in the previous section, not all the Hilbert functions allowed by 3 -codimensional aG schemes are available on $S$. For instance let $H$ be an $O$-sequence satisfying the Stanley conditions in order to be the Hilbert function of a 3 -codimensional aG subscheme of $\mathbb{P}^{3}$, where $s=\operatorname{deg} S=$ $\min \left\{i \mid \Delta^{r-1} H(i)<i+1\right\}$.

1. On our general hypersurface $S$ there is no 3 -codimensional aG schemes $X$ such that $H_{X}=H$ and $s>\Delta^{r-1} H(t)=\Delta^{r-1} H(t+1)>0$, for some $t$. This follows since by Theorem 3.1 in [RZ] in such a case $S$ should be reducible.

2. On our general hypersurface $S$ there is no 3 -codimensional aG scheme $X$, not complete intersection, such that $H_{X}=H$ and $\Delta^{r-1} H(t)=$ $\cdots=\Delta^{r-1} H(t+u-1)=0>\Delta^{r-1} H(t+u)$, for some $u \geq 2$. This follows since by a result of Iarrobino and Kanev in [IK] or by Corollary 3.3 in [RZ] one sees that such an $X$ should lie on a 2-codimensional aCM scheme contained in $S$ and which is not complete intersection of $S$.

This section is devoted to find other restrictions on a sequence $H$ in order to be a possible Hilbert function of a 3-codimensional aG scheme on a general hypersurface $S$. 
Proposition 3.1. Let $X \subset \mathbb{P}^{r}$ be a 3 -codimensional a $G$ scheme and $t \in \mathbb{N}$ such that $\Delta^{r} H_{X}(t)=-h<0, \Delta^{r} H_{X}(t+1)=-k<0$, with $h>k$, and $t+1 \leq \gamma=\min \left\{i \mid \Delta^{r-1} H_{X}(i) \leq 0\right\}$. Then there exists a $(2 m+1) \times(2 m+1)$ alternating matrix associated to $X$ of the following form:

$$
\left(\begin{array}{c|c|c}
* & * & A \\
\hline * & U & 0 \\
\hline-{ }^{t} A & 0 & 0
\end{array}\right)
$$

where $A$ is a $(\rho+k+1) \times \rho$ matrix and $U$ is an alternating matrix of order $2(m-\rho)-k$, with $m>\rho$.

Proof. By the assumptions we have the following situation

\begin{tabular}{c|ccccccc}
$n$ & 0 & 1 & $\cdots$ & $t-1$ & $t$ & $t+1$ & $\cdots$ \\
\hline$\Delta^{r-1} H_{X}$ & 1 & 2 & $\cdots$ & $c+h+k$ & $c+k$ & $c$ & $\cdots$ \\
\hline$\Delta^{r} H_{X}$ & 1 & 1 & $\cdots$ & $\cdots$ & $-h$ & $-k$ & $\cdots$ \\
\hline$\Delta^{r+1} H_{X}$ & 1 & 0 & $\cdots$ & $\cdots$ & $\cdots$ & $h-k>0$ & $\cdots$
\end{tabular}

Let $\rho$ be the number of first syzygies of $I_{X}$ of degrees less than or equal to $t+1$. Since $\Delta^{r+1} H_{X}(j)=-\alpha_{j}+\beta_{j}, h-k>0$ will imply $\rho>0$. If $\sigma$ denotes the number of minimal generators of $I_{X}$ of degrees less than or equal to $t+1$, we have $1+\rho-\sigma=-k$, i.e. $\sigma=1+\rho+k$. This shows that there are $\rho$ syzygies just acting on at most $\rho+k+1$ generators. Therefore an alternating matrix associated to $X$ will have the last $\rho$ columns as in the matrix (1). On the other hand, if we denote by $\vartheta$ the degree of the second syzygy of $I_{X}$, by the assumption $t+1 \leq \gamma$, we have $t+1<\vartheta / 2$, therefore $I_{X}$ must have at least $\rho$ more generators (of degrees bigger than $t+1)$, hence $2 \rho+k+1 \leq 2 m+1$; then $\rho \leq m-k / 2<m$.

Now we will apply the previous result to give stronger restrictions on the Hilbert functions of 3-codimensional subschemes of a general hypersurface of $\mathbb{P}^{r}$.

For this we need a result of linear algebra which can be probably deduced from general results of Buchsbaum and Eisenbud (see [BE]). Anyway, for reader's convenience, here we give a sketch of the proof.

Lemma 3.2. Let $A=\left(a_{i j}\right)$ be an alternating matrix of order $2 n, a_{i j} \in$ $R, r \leq n$ any positive integer and $s=2 n-r$. Suppose that for every 
$(i, j) \geq(s, s) a_{i j}=0$. Then

$$
\operatorname{Pf}(A)=\sum_{1 \leq i_{1}<\cdots<i_{r} \leq s} M_{i_{1} \cdots i_{r}} \operatorname{Pf}_{i_{1} \cdots i_{r}}
$$

where $M_{i_{1} \ldots i_{r}}$ is the minor of $A$ associated to the rows $i_{1}, \ldots, i_{r}$ and to the last $r$ columns and $\mathrm{Pf}_{i_{1} \cdots i_{r}}$ denotes the pfaffian of the alternating matrix of order $2(n-r)$ obtained from $A$ deleting the lines $i_{1}, \ldots, i_{r}$ and the last $r$ lines.

Proof. We use induction on $r$. The case $r=1$ is well known; in the general case we compute $\operatorname{Pf}(A)$ applying the result for $r=1$ obtaining

$$
\operatorname{Pf}(A)=\sum_{i}(-1)^{i} a_{i 2 n} \operatorname{Pf}_{i}
$$

Observe that $\mathrm{Pf}_{i}$ are pfaffians of alternating matrices of order $2 n-2$ which, by induction, can be computed by using the last $r-1$ columns. Now a simple but tedious computation leads to the required result.

Theorem 3.3. Let $X \subset \mathbb{P}^{r}$ be a 3-codimensional a $G$ scheme and $H=$ $H_{X}$ its Hilbert function, $S$ an hypersurface of minimal degree containing $X$ and $I_{S}=(f)$. If there exists an integer $t+1<\vartheta / 2$ such that $\Delta^{r} H(t)=$ $-h<\Delta^{r} H(t+1)=-1$, then either $S$ is reducible or there exists a 2codimensional aCM scheme $C$ such that $f \in I_{C}$ and $f$ is not a minimal generator for it.

Proof. Using Proposition 3.1, by ordering the minimal generators of $I_{X}$ in not decreasing degrees and the first syzygies in not increasing degrees, we can associate to $X$ an alternating matrix $M$ of type (1) with $k=1$. Therefore, the form $f$ defining $S$ is the pfaffian of the submatrix $N$ of $M$ obtained by deleting the first row and the first column:

$$
N=\left(\begin{array}{c|c|c}
* & * & B \\
\hline * & U & 0 \\
\hline-{ }^{t} B & 0 & 0
\end{array}\right)
$$

where $B$ is a $(\rho+1) \times \rho$ matrix. Now, we compute this pfaffian by applying Lemma 3.2 to the last $\rho$ columns. Since the only non zero minors of such a submatrix are those of $B$, if we denote by $J$ the ideal generated by these minors, we have $f \in J$. If $h t(J)=1$ then $S$ is reducible; otherwise, by the Hilbert-Burch Theorem, $J$ will be the saturated homogeneous ideal of a 2-codimensional aCM scheme for which $f$ is not a minimal generator. 
Corollary 3.4. Let $X \subset \mathbb{P}^{r}$ be a 3-codimensional aG scheme, with Hilbert function $H$, and let suppose that an hypersurface of minimal degree containing $X$ is general. Then, if for some integer $t<\vartheta / 2-1 \Delta^{r} H(t+1)=$ -1 then $\Delta^{r} H(t) \geq-1$.

For some particular Hilbert functions with the previous conditions we can give more information on the ideal of the scheme $X$ and consequently on the hypersurface of minimal degree containing $X$.

So let $X \subset \mathbb{P}^{r}$ be a 3 -codimensional aG scheme minimally generated by $f_{0}, f_{1}, \ldots, f_{2 m}, d_{0}=\operatorname{deg} f_{0} \leq d_{1}=\operatorname{deg} f_{1} \leq \cdots \leq d_{2 m}=\operatorname{deg} f_{2 m}$. Recall that $d_{m+1}+d_{m+2} \geq \vartheta$ implies that $\left(f_{0}, f_{1}, \ldots, f_{m}\right)$ is the saturated homogeneous ideal of a 2-codimensional aCM scheme (see [RZ], Proposition 3.5).

In the following proposition we will use this notation: $q=\left\lceil\frac{\vartheta-1}{2}\right\rceil$, $p=\max \left\{i \leq q \mid \Delta^{r} H_{X}(i) \neq-2\right\}$.

Proposition 3.5. Let $X \subset \mathbb{P}^{r}$ be a 3-codimensional aG scheme with Hilbert function $H$. If either $\Delta^{r} H_{X}(q) \geq-1$ or $\Delta^{r} H_{X}(q)=-2$ and $\Delta^{r} H_{X}(p) \geq-1$ then $d_{m+1}+d_{m+2} \geq \vartheta$.

Proof. Let $Y \subset \mathbb{P}^{r}$ be an aG scheme such that $H_{Y}=H$ with the minimal number of generators allowed by $H$ (such a scheme there exists, see for instance $[\mathrm{Di}]$ or $[\mathrm{GM}])$. Let $d_{0}^{\prime} \leq d_{1}^{\prime} \leq \cdots \leq d_{2 h}^{\prime}$ be the degrees of a minimal set of generators for $I_{Y}$. Of course $h \leq m$ and $d_{i}^{\prime} \in\left\{d_{0}, d_{1}, \ldots, d_{2 m}\right\}$ for $0 \leq i \leq 2 h$. We prove first that $d_{h+1}^{\prime}+d_{h+2}^{\prime} \geq \vartheta$. For convenience we set $b=\Delta^{r-1} H_{Y}(q-1)$ and $a=\Delta^{r-1} H_{Y}(q-2)$.

If $u \geq 2$ the conclusion follows by Corollary 3.4 in [RZ].

If $u=1$ and $\Delta^{r} H_{Y}(q)=-1$, i.e. $b=1$, using Lemma 3.6 in [RZ] we have:

$$
2 h+1=x+y+|a-2|+1
$$

where $x$ is the number of minimal generators of $Y$ of degree $<q$ and $y$ the number of minimal first syzygies of $I_{Y}$ of degree $<q$. Since $\Delta^{r} H_{Y}(q-1)=$ $1-a$ we have $1-x+y=1-a$. Combining this with the previous equality we get $x=h+\frac{a-|a-2|}{2}$. Therefore the number of minimal generators of $Y$ in degree $\leq q$ is $x+(2-a)_{+}=h+1$. Since $d_{h+1}^{\prime}$ is the degree of the $(h+2)-t h$ generator of $Y, d_{h+1}^{\prime}=q+1=\vartheta / 2$ (recall that when $u=1 \vartheta$ is even and in degree $\vartheta / 2$ there is just one generator, see [RZ] Lemma 3.6 or Remark 3.8). Therefore $d_{h+1}^{\prime}+d_{h+2}^{\prime}>\vartheta / 2+\vartheta / 2=\vartheta$. 
If $u=1, \Delta^{r} H_{Y}(q)=-2$ and $\Delta^{r} H_{Y}(p) \geq-1$ then $b=2$ and analogous computation shows that the number of minimal generators of $Y$ in degree $\leq q$ is $h+2$. Since $\Delta^{r} H_{Y}(p) \geq-1$ we have $\Delta^{r+1} H_{Y}(p+1)<0$ hence $d_{h+1}^{\prime}=p+1$ and $d_{h+2}^{\prime}>\vartheta-(p+1)$; consequently $d_{h+1}^{\prime}+d_{h+2}^{\prime}>\vartheta$.

When $u=0$ our hypotheses imply that $\Delta^{r} H_{Y}(q)=-2($ i.e. $b=1)$ therefore a similar computation as before says that $d_{h+1}^{\prime}=p+1$ and $d_{h+2}^{\prime}>$ $\vartheta-(p+1)$, hence $d_{h+1}^{\prime}+d_{h+2}^{\prime}>\vartheta$.

Now we will show that $d_{h+1}^{\prime}+d_{h+2}^{\prime} \geq \vartheta$ implies $d_{m+1}+d_{m+2} \geq \vartheta$. Observe first that we can obtain the $(2 m+1)$-tuple $\left(d_{0}, d_{1}, \ldots, d_{2 m}\right)$ from the $(2 h+1)$-tuple $\left(d_{0}^{\prime}, d_{1}^{\prime}, \ldots, d_{2 h}^{\prime}\right)$ by adding $m-h$ pairs of the type $(\delta, \vartheta-\delta)$, with $\delta \leq \vartheta-\delta$, see Remark 3.8 in [RZ]. Of course, by induction, we need just to prove the conclusion when we add to $\left(d_{0}^{\prime}, d_{1}^{\prime}, \ldots, d_{2 h}^{\prime}\right)$ a pair. Let us denote by $\left(e_{0}, e_{1}, \ldots, e_{2 k}\right), k=h+1,\left(e_{0} \leq e_{1} \leq \cdots \leq e_{2 k}\right)$ the $(2 k+1)$-tuple obtained by adding the pair $(\delta, \vartheta-\delta)$; we need to prove that $e_{k+1}+e_{k+2} \geq \vartheta$. If $\delta \geq d_{h+1}^{\prime}$ we get that $e_{k+1} \geq d_{h+1}^{\prime}$ and $e_{k+2} \geq d_{h+2}^{\prime}$ so the conclusion follows. If $\delta \leq d_{h+1}^{\prime}$ and $\vartheta-\delta \geq d_{h+2}^{\prime}$ we have that $e_{k+1}=d_{h+1}^{\prime}$ and $e_{k+2}=d_{h+2}^{\prime}$ and again we are done. Finally when $\delta \leq d_{h+1}^{\prime}$ and $\vartheta-\delta \leq d_{h+2}^{\prime}$ then $e_{k+1}+e_{k+2}=d_{h+1}^{\prime}+\vartheta-\delta \geq \vartheta$.

The next example shows that the previous result cannot be reversed in general.

Example 3.6. Consider a 3 -codimensional aG subscheme $X$ of $\mathbb{P}^{r}$ whose minimal generators' degrees are

$$
5,6,7,7,8,9,9
$$

(note that such a subscheme there exists since the Diesel's conditions are satisfied, see [Di] Proposition 3.1). For this scheme it is $d_{m+1}+d_{m+2}=$ $8+9 \geq 17=\vartheta$. Nevertheless $\Delta^{r} H_{X}(q)=-2$ and $\Delta^{r} H_{X}(p)=-3$ (in this case $q=8$ and $p=7$ ).

We want to prove that the previous result can be reversed for the schemes with a minimal number of generators allowed by their Hilbert function.

Proposition 3.7. Let $X \subset \mathbb{P}^{r}$ be as in the Proposition 3.5 with the minimal number of generators allowed by its Hilbert function $H$. Then $d_{m+1}+d_{m+2} \geq \vartheta$ if and only if $\Delta^{r} H(q) \geq-1$ or $\Delta^{r} H(q)=-2$ and $\Delta^{r} H(p) \geq-1$. 
Proof. The only if part is the Proposition 3.5.

Now suppose $\Delta^{r} H(q) \leq-2$ and, when $\Delta^{r} H(q)=-2, \Delta^{r} H(p)<-2$ (the inequality is strict by definition of $p$ ). Using the same notation as before, this implies immediately that $u<2$.

If $u=1$ (which implies $\vartheta=2 q+2$ ) and $\Delta^{r} H(q)<-2$, with the same computation done in the proof of Proposition 3.5 we see that the number of generators of degree $\leq q$ is bigger than or equal to $m+2$. Therefore $d_{m+1}+d_{m+2} \leq q+q+1=2 q+1<\vartheta$. If $\Delta^{r} H(q)=-2$ and $\Delta^{r} H(p)<-2$, we see that $d_{m+1} \leq p$ and $d_{m+2}=\vartheta-(p+1)$; so, $d_{m+1}+d_{m+2} \leq \vartheta-1<\vartheta$.

If $u=0$ we observe first that $\Delta^{r} H(p+1)>0$ and $\Delta^{r+1} H(i)=0$ for $p+2 \leq i \leq q$; thus the number of generators of degree $\leq q$ coincides with the number of generators of degree $\leq p$ and it is $m+1+b$. Hence $d_{m+1} \leq p$ and $d_{m+2} \leq \vartheta-(p+1)$, therefore $d_{m+1}+d_{m+2} \leq p+\vartheta-(p+1)<\vartheta$.

\section{$\S 4$. Betti numbers on general hypersurfaces}

In the previous section we saw as the "generality" of the hypersurface $S$ affects the postulation of the 3 -codimensional aG schemes which are on $S$. A similar question can be posed for the Betti numbers of such schemes. Precisely if $H$ is the Hilbert function of a 3-codimensional aG scheme $X$ on a general hypersurface $S \subset \mathbb{P}^{r}$, what Betti numbers allowed by $H$ according to Diesel conditions can occur in such an hypersurface?

The next result is a very beginning step on this direction.

Proposition 4.1. Let $S \subset \mathbb{P}^{r}$ be a general hypersurface with $\operatorname{deg} S=$ $d_{0}$. If $X \subset S$ is a 3 -codimensional a $G$ scheme with

$$
H_{X}=H_{Z}
$$

where $Z$ is some complete intersection of type $\left(d_{0}, d_{1}, d_{2}\right), d_{0} \leq d_{1} \leq d_{2}$, then $X$ is complete intersection.

Proof. Let $f_{0}, f_{1}, f_{2}$ be the generators of $I_{X}$ of degrees respectively $d_{0}, d_{1}, d_{2}$; let $d$ be the least degree of a minimal generator of $I_{X}$ different from $f_{0}, f_{1}, f_{2}$. Note that since $\Delta^{r+1} H_{X}(j)=-\alpha_{j}+\beta_{j}$, we must have also a syzygy of degree $d$. If $d<d_{2}$, such a syzygy acts only on $f_{0}$ and $f_{1}$, hence $S$ should be reducible. Therefore all the generators of $I_{X}$ other than $f_{0}, f_{1}$, $f_{2}$ should have degrees bigger or equal to $d_{2}$. Recalling that we can get all the degrees of the minimal generators of $I_{X}$ by adjoing to $\left\{d_{0}, d_{1}, d_{2}\right\}$ pairs 
of type $(d, \vartheta-d),\left(\vartheta=d_{0}+d_{1}+d_{2}\right)$, we see that the generators' degrees for $I_{X}$ should be

$$
d_{0} \leq d_{1} \leq d_{2} \leq h_{1} \leq h_{2} \leq \cdots \leq h_{r} \leq \vartheta-h_{r} \leq \cdots \leq \vartheta-h_{2} \leq \vartheta-h_{1} .
$$

$I_{X}$ has $2 m+1$ generators with $m=r+1$ and in this sequence of integers in the places $m+2$ and $m+3$ we find $h_{r}$ and $\vartheta-h_{r}$, respectively. Therefore applying Proposition 3.5 in [RZ], we get that the first $m+1$ generators of $I_{X}$ generate a 2 -codimensional aCM scheme (not complete intersection) on $S$, a contradiction.

COROLlary 4.2. Let $S \subset \mathbb{P}^{r}$ be an irreducible hypersurface with $\operatorname{deg} S=d_{0}$. If $X \subset S$ is a 3 -codimensional a $G$ scheme with

$$
H_{X}=H_{Z}
$$

where $Z$ is some complete intersection of type $\left(d_{0}, d_{1}, d_{2}\right), d_{0} \leq d_{1} \leq d_{2}$, then the only generators of $I_{X}$, other the three generators in degrees $d_{0}, d_{1}$, $d_{2}$, can occur in degrees $j$ with $d_{2} \leq j \leq d_{0}+d_{1}$.

Corollary 4.3. Let $S \subset \mathbb{P}^{r}$ be an irreducible hypersurface with $\operatorname{deg} S=d_{0}$. If $X \subset S$ is a 3 -codimensional a $G$ scheme with

$$
H_{X}=H_{Z}
$$

where $Z$ is some complete intersection of type $\left(d_{0}, d_{1}, d_{2}\right), d_{2} \geq d_{0}+d_{1}$, then $X$ is a complete intersection.

Proof. The only case which is not consequence of the previous corollary is when $d_{2}=d_{0}+d_{1}$; but in this situation $\Delta^{r} H_{X}\left(d_{2}\right)=-1$ therefore $I_{X}$ has only one generator in degree $d_{2}$ (see [RZ] Proposition 3.7).

Recall that if $X \subset \mathbb{P}^{r}$ is a 3-codimensional aG scheme, with Hilbert function $H$, if $\alpha_{i}$ denotes the number of minimal generators of $I_{X}$ in degree $i$, by Proposition 3.7 in [RZ] or Proposition 1.2 in [CV], there are sharp bounds for $\alpha_{i}$ depending only on $H$, precisely, for $s+1 \leq i \leq \vartheta-s-1$

$$
m_{i} \leq \alpha_{i} \leq M_{i}
$$

where $M_{i}=-\Delta^{r} H(i)$ and

$$
m_{i}= \begin{cases}1, & \text { for } u=1, \Delta^{r-1} H\left(\frac{\vartheta}{2}-2\right) \text { odd }, i=\vartheta / 2 \\ \max \left\{0,-\Delta^{r+1} H(i)\right\}, & \text { otherwise }\end{cases}
$$


where $s=\min \left\{i \in \mathbb{N} \mid\left(I_{X}\right)_{i} \neq 0\right\}$ (of course $\alpha_{s}=-\Delta^{r+1} H(s)$ ).

The next result will deal with some geometrical consequences for schemes for which $\alpha_{n}=M_{n}>m_{n}$ for some degree $n$.

TheOREM 4.4. Let $X \subset \mathbb{P}^{r}$ be a 3-codimensional aG scheme, with Hilbert function $H$. Suppose that there exists an integer $n, s+1 \leq n \leq$ $\vartheta-s-1$ such that $\alpha_{n}=M_{n}>m_{n}$ and let $J=\left(I_{X}\right)_{\leq n-1}$. Then there exists a form $g, \operatorname{deg} g=\left|\Delta^{r-1} H(n-1)\right|$, such that $J \subset(g)$ and $J: g$ is the saturated homogeneous ideal of a 2-codimensional aCM subscheme of $\mathbb{P}^{r}$.

Proof. Of course, by Remark 3.8 of [RZ], we can suppose $n \leq \vartheta / 2$. Let $\rho$ be the number of syzygies of $I_{X}$ of degree $\leq n$ and $\sigma$ the number of generators of degree $\leq(n-1)$. By assumption, we have $\alpha_{n}=-\Delta^{r} H(n)>0$ and the number of syzygies in degree $n$ is $\beta_{n}=\Delta^{r+1} H(n)-\Delta^{r} H(n)>0$. Then,

$$
1+\rho-\sigma=\Delta^{r} H(n-1)+\beta_{n}=\Delta^{r} H(n-1)+\Delta^{r+1} H(n)-\Delta^{r} H(n)=0 .
$$

This shows, as in the Theorem 3.1 in [RZ], that an alternating matrix associated to $X$ has the following shape

$$
\left(\begin{array}{c|c|c}
* & * & A \\
\hline * & U & 0 \\
\hline-{ }^{t} A & 0 & 0
\end{array}\right)
$$

where $A$ is a $(\rho+1) \times \rho$ matrix and $U$ is an alternating matrix of order $2(m-\rho),\left(2 m+1=\nu\left(I_{X}\right)\right)$. Now, if $g$ is the pfaffian of $U$, we see that all the $\rho+1$ generators of degree less or equal to $n-1$ will have $g$ as factor.

Observe that for $0 \leq i \leq \rho$ the generators of $I_{X}$ will have the form $f_{i}=g_{i} g$, where $g_{i}$ are the maximal minors of the matrix $A$. Since $X$ is a 3 -codimensional aG scheme the matrix $A$ is the Hilbert-Burch matrix of a 2-codimensional aCM scheme. In fact, an easy linear algebra computation, that one can find also for instance in the proof of Theorem 5.14 in [IK], shows that the maximal minors of $A$ cannot have a common factor of positive degree. This shows that $J \subset(g)$ and $J: g$ is a 2-codimensional aCM scheme. Now, to compute $\operatorname{deg} g$, we set $d_{i}=\operatorname{deg} f_{i}$ and $e_{i}$ the degrees of the $\rho$ syzygies of degree less or equal to $n$; we have

$$
-\sum_{i=0}^{\rho}\left(d_{i}-\operatorname{deg} g\right)+\sum_{j=1}^{\rho}\left(e_{j}-\operatorname{deg} g\right)=0
$$


which implies

$$
\begin{aligned}
\operatorname{deg} g & =\sum_{i=0}^{\rho} d_{i}-\sum_{j=1}^{\rho} e_{j}=-\sum_{i=0}^{n-1} i \Delta^{r+1} H(i)-n \beta_{n} \\
& =-(n-1) \Delta^{r} H(n-1)+\Delta^{r-1} H(n-2)-n\left(\Delta^{r+1} H(n)-\Delta^{r} H(n)\right) \\
& =\Delta^{r} H(n-1)+\Delta^{r-1} H(n-2)=\Delta^{r-1} H(n-1)
\end{aligned}
$$

(note that $\Delta^{r-1} H(n-1) \geq 0$ since we are working now with $n \leq \vartheta / 2$ ).

Remark 4.5. Observe that in the hypothesis of the previous theorem the only case in which $\operatorname{deg} g=0$ occurs when $u=1$ and $n=\vartheta / 2$; in fact, $\Delta^{r-1} H(n-1)=0$ implies $u \geq 1$ and, on the other hand $M_{n}>m_{n}$ implies $u \leq 1$. Thus, $u=1, \vartheta$ is even and the only 0 in $\Delta^{r-1} H$ occurs in $\vartheta / 2-1$. So, a 3-codimensional aG scheme on an irreducible hypersurface of minimal degree cannot have $\alpha_{n}=M_{n}>m_{n}$ in all cases except when $u=1$ and $n=\vartheta / 2$.

Corollary 4.6. Let $X \subset \mathbb{P}^{r}$ be a 3-codimensional aG scheme, with Hilbert function $H$, contained in a hypersurface of minimal degree $S$.

If $S$ is general then for any $n \in \mathbb{N}$ in which $M_{n}>m_{n}, \alpha_{n}<M_{n}$.

If $S$ is irreducible the same is true except when $u=1$ and $n=\vartheta / 2$.

Theorem 4.7. Let $X \subset \mathbb{P}^{r}$ be a 3-codimensional aG scheme, with Hilbert function $H, S$ an hypersurface of minimal degree containing $X$ and $I_{S}=(f)$. Suppose that there exists an integer $n, s+1 \leq n \leq \vartheta-s-1$ such that $\alpha_{n}=M_{n}-1>m_{n}$. Then, either $S$ is reducible or there exists a 2-codimensional aCM scheme $C$ such that $f \in I_{C}$ for which $f$ is not a minimal generator.

Proof. Of course, we can suppose again $n \leq \vartheta / 2$. Let $\rho$ be the number of syzygies of $I_{X}$ of degree $\leq n$ and $\sigma$ the number of generators of degree $\leq n-1$. By assumption, we have $\alpha_{n}=-\Delta^{r} H(n)-1>0$ and the number of syzygies in degree $n$ is $\beta_{n}=\Delta^{r+1} H(n)-\Delta^{r} H(n)-1>0$. Then,

$1+\rho-\sigma=\Delta^{r} H(n-1)+\beta_{n}=\Delta^{r} H(n-1)+\Delta^{r+1} H(n)-\Delta^{r} H(n)-1=-1$.

This shows, as in the Theorem 3.1 in [RZ], that an alternating matrix associated to $X$ has the following shape

$$
\left(\begin{array}{c|c|c}
* & * & A \\
\hline * & U & 0 \\
\hline-{ }^{t} A & 0 & 0
\end{array}\right)
$$


where $A$ is a $(\rho+2) \times \rho$ matrix and $U$ is an alternating matrix of order $2(m-\rho),\left(2 m+1=\nu\left(I_{X}\right)\right)$. Therefore, the form $f$ defining $S$ is the pfaffian of the submatrix $N$ of $M$ obtained by deleting the first row and the first column:

$$
N=\left(\begin{array}{c|c|c}
* & * & B \\
\hline * & U & 0 \\
\hline-{ }^{t} B & 0 & 0
\end{array}\right)
$$

where $B$ is a $(\rho+1) \times \rho$ matrix. Now, the same argument as Theorem 3.3 will show that $f$ belongs to the ideal $J$ generated by the maximal minors of $B$. Therefore, if $h t(J)=1 S$ is reducible; otherwise, $J$ will be the saturated ideal of a 2-codimensional aCM scheme.

Corollary 4.8. Let $X \subset \mathbb{P}^{r}$ be a 3-codimensional aG scheme, with Hilbert function $H$, and let suppose that an hypersurface of minimal degree containing $X$ be general. Then, for every $n$

$$
\left\{\begin{array}{l}
M_{n} \leq m_{n}+1 \Longrightarrow \alpha_{n}=m_{n} \\
M_{n} \geq m_{n}+2 \Longrightarrow m_{n} \leq \alpha_{n} \leq M_{n}-2 .
\end{array}\right.
$$

Proof. Just use the previous theorem, reminding that $S$ is irreducible and does not contain 2-codimensional aCM schemes except complete intersections of type $S \cap T$.

We would like to conclude by asking the following questions:

1. Construct examples of 3-codimensional aG subschemes of $\mathbb{P}^{r}$, contained in a general hypersurface of minimal degree, for which $\alpha_{n}>m_{n}$ for some $n$.

2. Are there hypersurfaces $S$ containing only 3 -codimensional aG schemes with $\alpha_{n}=m_{n}$ for every $n$ for which $S$ is of minimal degree?

\section{REFERENCES}

[BE] D. A. Buchsbaum and D. Eisenbud, Algebra structures for finite free resolutions, and some structure theorems for ideals of codimension 3, Amer. J. Math., 99 (1) (1977), 447-485.

[CV] A. Conca and G. Valla, Betti numbers and lifting of Gorenstein codimension three ideals, preprint.

[DV] E. De Negri and G. Valla, The h-vector of a Gorenstein codimension three domain, Nagoya Math. J., 13 (1995), 113-140. 
[Di] S. Diesel, Irreducibility and dimension theorems for families of height 3 Gorenstein algebras, Pac. J. of Math., 172 (4) (1996), 365-397.

[GM] A. Geramita and J. Migliore, Reduced Gorenstein codimension three subschemes of projective space, Proc. Amer. Math. Soc., 125 (4) (1997), 943-950.

[Ha] T. Harima, Some examples of unimodal Gorenstein sequences, J. Pure Appl. Algebra, 103 (1995), 313-324.

[HTV] J. Herzog, N. V. Trung and G. Valla, On hyperplane sections of reduced irreducible varieties of low codimension, J. Math. Kyoto Univ., 34 (1) (1994), 47-72.

[IK] A. Iarrobino and V. Kanev, The length of a homogeneous form, determinantal loci of catalecticants and Gorenstein algebras, preprint.

[RZ] A. Ragusa and G. Zappalà, Properties of 3-codimensional Gorenstein schemes, to appear Comm. in Alg.

[St] R. Stanley, Hilbert functions of graded algebras, Adv. in Math., 28 (1978), 57-83.

[Ul] B. Ulrich, Sums of linked ideals, Trans. Am. Math. Soc., 318 (1990), 1-42.

\author{
Alfio Ragusa \\ Dipartimento di Matematica \\ Università di Catania \\ Viale A. Doria 6 \\ 95125 Catania \\ Italy \\ ragusa@dipmat.unict.it \\ Giuseppe Zappalà \\ Dipartimento di Matematica \\ Università di Catania \\ Viale A. Doria 6 \\ 95125 Catania \\ Italy \\ zappalag@dipmat.unict.it
}

